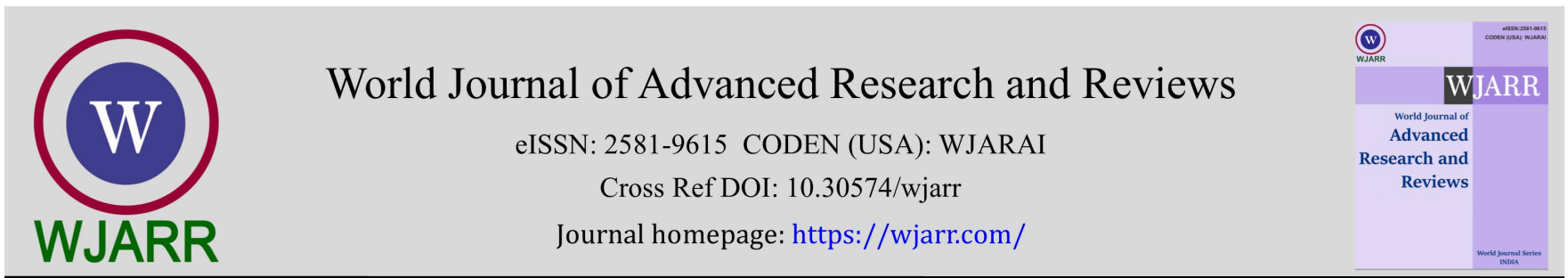

(RESEARCH ARTiCle)

Check for updates

\title{
The effects of Kinesio taping-combined exercise on the balance and gait ability of hemiplegic patients
}

\author{
Young-Han Park ${ }^{1}$, Dae-Hwan Lee ${ }^{2}$ and Youn-Bum Sung 2,* \\ 1 Department of Physical Therapy, Korea National University of Transportation, 61, Daehak-ro, Jeungpyeong-eup, \\ Jeungpyeong-gun, Chungcheongbuk-do, Republic of Korea. \\ 2 Department of Physical Therapy, Daegu University, Jillyang-eup, Gyeongsan-si, Gyeongsangbuk-do, Republic of Korea.
}

World Journal of Advanced Research and Reviews, 2021, 12(03), 322-328

Publication history: Received on 10 November 2021; revised on 14 December 2021; accepted on 16 December 2021

Article DOI: https://doi.org/10.30574/wjarr.2021.12.3.0700

\begin{abstract}
Purpose: This study aims to investigate the effect of the Kinesio taping method on the balance ability and gait ability of hemiplegic stroke patients by applying it to the lower extremities of the ankle on the affected side, addressing instability and asymmetry by improving the stability of the muscles and ankle joint.
\end{abstract}

Methods: This study confirmed the general characteristics of hemiplegic patients diagnosed with stroke. Kinesio taping was applied to the lower extremities of 15 subjects in the experimental group, and they performed mat and treadmill exercises. In addition, basic mat and treadmill exercises were performed by the 15 subjects in the control group. The exercise regimens were performed 18 times: three times a week for six weeks.

Results: In the paired-sample T-test used for within-group comparison of BBS, TUG and stance time on nonparetic side value, there was a significant difference between pre- and post-test for experimental group. However, in the control group, there was a statistically significant difference only in TUG. In the inter-group comparison, the experimental group showed a statistically significant improvement in BBS, TUG and stance time on nonparetic side value compared to the control group.

Conclusion: The experiment's results demonstrate that the application of Kinesio taping-combined exercise provides stability of the muscles and ankle joints of the lower extremities during walking by improving balance ability, and improves overall gait stability by increasing the stance phase time of the affected side in hemiplegic stroke patients.

Keywords: Kinesio taping, Balance, Gait, Rehabilitation, Stroke

\section{Introduction}

Stroke is a leading cause of death worldwide and one of the major diseases causing physical dysfunctions in adults. According to Statistics Korea, the death rate due to stroke was 53.2 per 100,000 people, and stroke is the second leading cause of death after cancer. Moreover, as a single organ disease, it is the number one cause of death. The incidence of stroke in Korea is much higher than in other OECD countries, and this is expected to triple by 2030, due to the aging Korean population. Therefore, effective stroke prevention and treatment programs are urgently required [1].

Stroke is defined as a syndrome with consciousness and motor disorders that causes blood circulation problems by cutting off the blood supply to the brain. This happens when the blood flow to the brain is blocked or bleeding occurs

\footnotetext{
* Corresponding author: Youn-Bum Sung; E-mail:playeryoon@naver.com

Department of Physical Therapy, Daegu University, Jillyang-eup, Gyeongsan-si, Gyeongsangbuk-do, Republic of Korea.

Copyright (C) 2021 Author(s) retain the copyright of this article. This article is published under the terms of the Creative Commons Attribution Liscense 4.0.
} 
due to damage to the blood vessels. Currently, about two-thirds of strokes develop in patients in the 60 years or older age group, but it can develop at any age, including in young children. Stroke can be caused by hypertension, diabetes, heart disease, and arteriosclerosis. The probability of stroke increases in people who have previously experienced a stroke or have a family history of stroke. Common symptoms of stroke include hemiplegia, partial paralysis of the body, and numbness and loss of sensation in parts of the body. Furthermore, stroke patients not only experience physical disabilities, but also disorders in their cognitive, visual, language, and social abilities, and may show symptoms of paralysis in parts of the body and impairment of motor ability that limits movement in their daily lives [2]. In addition, stroke patients show problems in maintenance of postural alignment, voluntary movement, and motion control, due to a combination of muscle weakness or muscle imbalance, and therefore, function ineffectively in physical activity. These symptoms may appear mild or temporary in the early stage of stroke and then disappear. However, if these symptoms are severe, a permanent disability, a decrease in physical function, and psychological depression are likely to occur [3].

Stroke is the most common neurological disease in the world, and among developed countries, it is the second most common disease and a leading cause of disability in adults. In rare cases, some stroke patients may show normal gait patterns, but most of them experience impaired gait. These limitations mainly occur on one side of the body, with the left and right sides of the body divided into normal and affected sides. Weight bearing on the normal side increases while weight bearing on the affected side decreases [4]. Furthermore, asymmetric postural alignment of the trunk and legs in hemiplegic patients degrades the stability function of the proximal part of the lower extremities and the trunk, making it impossible to perform the functions of normal and natural gait patterns. Hemiplegia, a common problem of stroke, interferes with the symmetrical balance of the body because the patient uses the normal side more than the affected side. Stroke patients support 30-40\% of their total body weight on the affected side while walking. This causes instability of body balance and postural control and leads to problems, such as a decrease in exercise and balance abilities [5,6].

Stability of the lower extremities is required for good gait, and ankle stability, in particular, plays a key role during walking. Kinesio taping can correct muscle function by fixing muscles and joints, correcting misalignment of joints, and improving exercise performance by preventing secondary damage; therefore, relieving pain and improving muscle strength, agility, muscular endurance, and flexibility. Moreover, it is used to improve body balance ability by controlling postural balance. Kinesio taping, which involves attaching an elastic tape to a specific region, can be used in various ways in daily life because it is relatively simple and easy to learn [7-9].

This study aims to investigate the effect of the Kinesio taping method on the balance ability and gait ability of hemiplegic stroke patients by applying it to the lower extremities of the ankle on the affected side, addressing instability and asymmetry by improving the stability of the muscles and ankle joint.

\section{Material and methods}

\subsection{Subjects}

This study involved 30 patients who were diagnosed with stroke and hospitalized at J Hospital in C City. Hemiplegic stroke patients without orthopedic disease in the trunk and both lower extremities, which could affect the treatment effect, were selected. The patients provided informed consent after the contents and purpose of this study were explained. In addition, the selected patients scored 21 or higher on the Korean Simple Mental State Discrimination Test (MMSE-K), indicating they were able to communicate and had the cognitive ability to understand and carry out instructions. They were then divided into an experimental group of 15 subjects and a control group of 15 subjects.

\subsection{Study design}

This study confirmed the general characteristics of hemiplegic patients diagnosed with stroke. Through random assignment by number lottery, odd-numbered subjects were assigned to the experimental group, and even-numbered subjects were assigned to the control group. Kinesio taping was applied to the lower extremities of 15 subjects in the experimental group, and they performed mat and treadmill exercises. In addition, basic mat and treadmill exercises were performed by the 15 subjects in the control group. The exercise regimens were performed 18 times: three times a week for six weeks. The same evaluation sheet was used for every subject and a video was taken while the subjects were walking. The data obtained through a pre-test before exercise and post-test after exercise were analyzed using the SPSS/Windows (version 20.0) statistical analysis program. 


\section{Evaluation method}

\subsection{Evaluation of balance ability}

The balance ability was evaluated by measuring the dynamic balance ability using the Berg Balance Scale (BBS). The BBS is used to clinically evaluate the balance ability of hemiplegic patients with geriatric disease or stroke in moving or standing positions. The scale consists of 14 items, and each item is scored between 0 and 4 , with a total score of 56 . The intra- and inter-rater reliability of this measurement tool were $r=.99$ and $r=.98$, respectively. Thus, it had high reliability and internal validity in evaluating balance ability [10].

The evaluation items consist of; sit to stand, standing without assistance, sitting on their own without leaning, stand to sit, moving, standing with eyes closed, standing with feet together, standing with arms outstretched, lifting objects off the floor while standing, looking back over both shoulders while standing, turning 360 degrees, alternating feet on foot while standing, standing unsupported with one foot in front of the other, and standing on one foot. The smaller the score is from 56, the lower the patient's balance ability and the higher the risk of falls become.

\subsection{Evaluation of gait ability}

\subsubsection{TUG (timed up \& go test) and Dartfish Program}

TUG is a test method that can quickly indicate motility and gait by measuring the time used to start from a sitting position with arms on a chair with armrests, walk a distance of $3 \mathrm{~m}$, come back, sit on the chair, and place arms on the armrests. It is performed three times and the average time is recorded. According to the research, it takes less than 10 seconds for adults with a normal sense of balance to perform TUG, and it takes 11 to 20 seconds for the frail elderly. If it takes more than 20 seconds, the risk of functional motor impairment and falls increases. This test is a reliable tool with an intra-rater reliability of 0.99 and an inter-rater reliability of 0.98 [11].

Another gait evaluation method, the Dartfish Program, was used after taking videos of subjects while they were walking. Spatiotemporal analysis methods are widely used to analyze the gait characteristics of hemiplegic patients. Particularly when a time factor is used, it has high clinical significance. The Dartfish Program was developed in 1998 by Dartfish, a company based in Switzerland. It is a software program that analyzes and calculates the motion trajectory of a subject using video recorded with software that observes and analyzes the movements of subjects of sports, biomechanics, and exercise therapy. For video measurement, the measurement sites of the subject, including the greater trochanter, the lateral epicondyle of the knee, the lateral malleolus of the ankle, and the metatarsophalangeal joint of the fifth toe, were marked with stickers [12]. Then the subjects were instructed to walk $5 \mathrm{~m}$ twice with the usual gait and, while they were walking, a video was recorded in the sagittal plane using a camera at a distance of $1 \mathrm{~m} \mathrm{[13].}$

\section{Treatment methods}

The experimental group performed the mat exercise first. The starting position was set by fixing and aligning the subjects' trunk position while they were lying down. Then after stretching the ankle and knee of the lower extremities, the therapist actively assisted with the movement of flexion and extension at the lower extremities joint of the subject. This exercise was performed for 10 minutes. In addition, an exercise of repeatedly sitting and standing on a chair, inducing pelvic movement, was performed for 10 minutes.

After applying Kinesio taping to the lower extremities, a gait exercise was performed using a treadmill. The treadmill was started at the minimum speed of $1 \mathrm{~km} / \mathrm{h}$ and this was gradually increased up to $2 \mathrm{~km} / \mathrm{h}$ to allow the subjects to adapt themselves without experiencing difficulty in walking. For safety, during the treadmill exercise, a safety clip was attached to the patient's clothes. The subjects were instructed to hold the left and right handles if they felt dizzy or lost balance during exercise. This exercise was performed for 20 minutes, three days a week for six weeks, and the subjects had a 2-3-minute rest between each exercise. The control group performed the same exercises as the experimental group, but without taping of their lower extremities[14,15].

The Kinesio taping method used, following that suggested by Seo et al. (2017), involved applying a piece of $5 \mathrm{~cm}$ wide Kinesio tape, starting on the outer malleolus and covering the malleolus. In the same way, another piece of tape was applied, starting on the outer malleolus, overlapping a third of the tape. An I-shaped tape was then applied, as if erecting a pillar on the ankle, starting from the heel and covering the ankle in a U-shape. A second I-shaped tape was applied, starting at the Achilles tendon and extending past the ankle bone in such a way that the two I-shaped tapes cross each other. When attaching the tapes so that they cross each other, the ankle was in a stretched position [9]. 


\section{Statistical analysis method}

The data obtained from the experiment were described by mean \pm standard deviation (Mean \pm SD), and statistical analysis was performed using SPSS/Windows (version 20.0). An independent sample t-test was performed for differences in the subjects' general characteristics. A paired-sample t-test was performed to examine the effect of intra-group change in each experimental and control group before and after the intervention. In addition, an independent sample t-test was performed to compare the inter-group change of the experimental and control groups. The statistical significance level was set to 0.05 .

\section{Results}

\subsection{General characteristics of subjects}

The mean age of the subjects was $67.80 \pm 9.337$ years for the experimental group and $67.67 \pm 6.543$ years for the control group. The mean weight of the subjects was $61.13 \pm 9.553 \mathrm{~kg}$ for the experimental group and $64.07 \pm 6.193 \mathrm{~kg}$ for the control group. The mean height of the subjects was $161.00 \pm 8.726 \mathrm{~cm}$ for the experimental group and $164.80 \pm 5.943 \mathrm{~cm}$ for the control group. The mean period of medical history was $28.27 \pm 11.087$ months for the experimental group and $30.20 \pm 9.937$ months for the control group. An independent sample t-test was performed for the subjects' general characteristics and the results are summarized in Table 1 . Statistically significant differences were not found ( $p>0.05$ ). Therefore, the experimental and control groups were homogeneous.

Table 1 General characteristics of the subject

\begin{tabular}{|l|l|l|l|l|}
\hline & EG(n=15) & CG(n=15) & t & p \\
\hline Age (years) & $67.80 \pm 9.337$ & $67.67 \pm 6.543$ & -0.045 & 0.964 \\
\hline Weight(kg) & $61.13 \pm 9.553$ & $64.07 \pm 6.193$ & 0.998 & 0.327 \\
\hline Height(cm) & $161.00 \pm 8.726$ & $164.80 \pm 5.943$ & 1.394 & 0.174 \\
\hline Time since onset of stroke (months) & $28.27 \pm 11.087$ & $30.20 \pm 9.937$ & 0.913 & 0.619 \\
\hline
\end{tabular}

\subsection{Pre and post results of BBS by group}

The experimental group showed a statistically significant difference in BBS with $34.266 \pm 3.692$ before the experiment and $38.733 \pm 3.369$ after the experiment $(\mathrm{p}<.05)$. However, the control group did not show a statistically significant difference with $35.733 \pm 3.654$ before the experiment and $37.133 \pm 5.139$ after the experiment ( $p>.05$ ). In the inter-group comparison, the experimental group showed a statistically significant improvement in balance ability compared to the control group $(\mathrm{p}<.05)$.

Table 2 The comparison of BBS value for each group at pre-test and post-test

\begin{tabular}{|l|l|l|l|l|}
\hline & EG(n=15) & CG(n=15) & t & p \\
\hline pre & $34.266 \pm 3.692$ & $35.733 \pm 3.654$ & & \\
\cline { 1 - 3 } post & $38.733 \pm 3.369$ & $37.133 \pm 5.139$ & \multirow{2}{*}{2.111} & \multirow{2}{*}{$0.044^{*}$} \\
\cline { 1 - 3 } $\mathrm{t}$ & -7.429 & -3.626 & & \\
\cline { 1 - 3 } $\mathrm{p}$ & $0.000^{*}$ & 0.106 & & \\
\hline \multicolumn{5}{|c|}{ BBS: Berg Balance Scale *: $\mathrm{p}<.05$ Results and discussion }
\end{tabular}

\subsection{Pre and post results of TUG by group}

In the experimental group, the TUG showed a statistically significant difference with $36.634 \pm 13.804$ before the experiment and 31.160 \pm 13.594 after the experiment $(\mathrm{p}<.05)$. Furthermore, the control group also showed a statistically significant difference with $37.333 \pm 10.708$ before the experiment and $35.666 \pm 10.641$ after the experiment ( $p>.05$ ). In the inter-group comparison, the experimental group showed a statistically significant improvement in TUG compared to the control group $(\mathrm{p}<.05)$. 
Table 3 The comparison of TUG value for each group at pre-test and post-test

\begin{tabular}{|c|c|c|c|c|}
\hline & $E G(n=15)$ & $C G(n=15)$ & $\mathbf{t}$ & $\mathbf{p}$ \\
\hline pre & $36.634 \pm 13.804$ & $37.333 \pm 10.708$ & \multirow{4}{*}{-2.246} & \multirow{4}{*}{$0.033^{*}$} \\
\hline post & $31.160 \pm 13.594$ & $35.666 \pm 10.641$ & & \\
\hline $\mathrm{t}$ & 3.623 & 5.801 & & \\
\hline $\mathrm{p}$ & $0.000^{*}$ & $0.000^{*}$ & & \\
\hline
\end{tabular}

\subsection{Pre and post results of stance time on nonparetic side value by group}

The experimental group showed a statistically significant change in stance time on nonparetic side value from $1.42 \pm .04$ before the experiment to $1.20 \pm .04$ after the experiment $(p<.05)$. However, the control group did not show a statistically significant change with $1.50 \pm .05$ before the experiment and $1.42 \pm .05$ after the experiment ( $>$ >05). In the inter-group comparison, the experimental group showed a statistically significant improvement in gait ability compared to the control group $(\mathrm{p}<.05)$.

Table 4 The comparison of stance time on nonparetic side value for each group at pre-test and post-test

\begin{tabular}{|c|c|c|c|c|}
\hline & $E G(n=15)$ & $\operatorname{CG}(n=15)$ & $\mathbf{t}$ & $\mathbf{p}$ \\
\hline pre & $1.42 \pm .04$ & $1.50 \pm .05$ & \multirow{4}{*}{-4.137} & \multirow{4}{*}{$0.000^{*}$} \\
\hline post & $1.20 \pm .04$ & $1.42 \pm .05$ & & \\
\hline $\mathrm{t}$ & 6.762 & 1.783 & & \\
\hline $\mathrm{p}$ & $0.000^{*}$ & 0.196 & & \\
\hline
\end{tabular}

\section{Discussion}

This study experimentally examined the effects of the Kinesio taping method on balance ability and gait ability, when applied to the ankle of lower extremities of the affected side of hemiplegic patients diagnosed with stroke, and the clinical usefulness of the Kinesio taping method. Stroke causes not only physical dysfunction but also cognitive dysfunction, which reduces functional independence and lowers social participation and life satisfaction, resulting in lower quality of life [16]. Moreover, stroke patients' decreased sensory function makes it difficult for them to maintain balance due to postural control, decreased joint movement ability, damage to proprioception, and weakening of muscle strength. Such abnormal body balance has a significant impact on the ability of stroke patients to maintain normal gait function and perform the activities of daily life, and it increases the risk of secondary damage, such as falls [17].

Balance disorders that increase the risk of falls interfere with stroke patients' motor functions and return to daily activities. Decreased balance ability interferes with independent gait by antagonizing contraction and relaxation of lower extremity muscles and causing sensory and kinesthetic disturbances on the affected side in stroke patients [18]. Furthermore, two-thirds of patients with stroke showed a decrease in motor ability accompanied by impairment in gait, and patients with lower extremity paralysis showed a decrease in walking speed due to problems with stance phase time and stride length. Therefore, balance control ability is an important factor in the performance of activities of daily living, and balance control learning is a basic goal of rehabilitation for stroke patients [19].

Taping is one of the methods used to help the body move by providing stability to the muscles and joints for balance control. The functional taping method is used for the control of muscle imbalance and unstable posture in the treatment and rehabilitation of motor injuries. Moreover, it shortens the treatment period of the injured area and prevents secondary joint or muscle damage by reinforcing the skin or ligaments [20]. Taping increases the muscle area transversely by stimulating the muscle spindle and tendon organs, and as the tape moves closer to the skin and tendon, it compresses these regions. Muscle spindles or tendon organs are activated by the delicate compression action on the muscles and tendons from above the skin, thus relieving muscle overtension, increasing muscle strength, and reducing pain [21]. The ultimate goal of taping therapy is to support or protect the joints so that they can perform efficient and 
best functional movements. External support by taping provides joint stability through reinforcement of ligaments and restriction of joint motion [22]. Previous studies reported that the taping effect on the gait of stroke patients stimulated proprioception and affected the stance phase during gait, which in turn influenced the stride distance, step length, and gait speed [7-9,13,20,23]. When the taping method was applied to the hip abductor muscle in 13 hemiplegic stroke patients who walked with a cane, increases in the activation of the abductor muscle and gait speed were observed, compared to general patients who walked with a cane. In another study, which investigated the effect of patellar taping using electromyography (EMG) in 14 women with patellofemoral pain, the subjects showed a difference in the activity of the vastus medialis muscle according to the position change when the vastus medialis and lateralis muscles were activated [24].

This study performed mat exercise and treadmill walking exercises for experimental and control groups to improve balance ability and gait ability. In addition, Kinesio taping was applied to the experimental group during exercise to investigate changes in balance ability and gait ability. For the balance ability evaluation in this study, the experimental and control groups were evaluated using BBS, which has high reliability and internal validity. In the experimental group, the changes before and after exercise showed statistically significant differences $(\mathrm{p}<.05)$, but the control group did not show statistically significant differences ( $p>.05)$. Furthermore, the inter-group comparison of changes showed a statistically significant difference $(\mathrm{p}<.05)$.

The gait ability was evaluated using TUG, which is a reliable tool with an intra-rater reliability of 0.99 and an inter-rater reliability of 0.98 . The Dartfish Program, as a program to observe and analyze the movement of the subjects of exercise therapy, was used to analyze the movement trajectory of the subjects using recorded videos to measure the time of the stance phase and swing phase. Both experimental and control groups showed statistically significant differences in the change of TUG before and after exercise $(\mathrm{p}<.05)$. The experimental group showed a statistically significant difference in stance phase time $(\mathrm{p}<.05)$, whereas the control group did not show a statistically significant difference in stance phase time $(\mathrm{p}<.05)$. The differences in change between the groups were statistically significant in BBS and TUG ( $<<.05)$.

The above results suggest that the Kinesio taping method used in this study can have a positive effect on balance ability and gait ability in hemiplegic stroke patients. However, some considerations are required during treatment. The patient may experience skin problems, erythema, and itching when Kinesio taping is applied. Furthermore, excessive pressure felt by the patient should be avoided, the tape should not be applied for a long time, and the tape should be removed immediately after exercise. The clinical relevance of the experimental results of this study should be evaluated in another study. This study also has a few limitations: the number of subjects is small, it is difficult to generalize when applying the intervention method, and it is difficult to predict the long-term treatment effect on stroke patients with a 6-week experiment.

\section{Conclusion}

The experiment's results demonstrate that the application of Kinesio taping-combined exercise provides stability of the muscles and ankle joints of the lower extremities during walking by improving balance ability, and improves overall gait stability by increasing the stance phase time of the affected side in hemiplegic stroke patients.

\section{Compliance with ethical standards}

\section{Acknowledgments}

This was supported by Korea National University of Transportation in 2021.

\section{Disclosure of conflict of interest}

No conflict of interest.

\section{Statement of informed consent}

Informed written consent were obtained from all individual participants included in the study

\section{References}

[1] Ji JG, \& Kwak YS. The combined effects of walking and kinesiotaping treatment on the serum inflammatory factors and lipid variables of stroke patients. 2017; Journal of Korean society for Wellness. 12(3): 591-602. 
[2] Duncan PW, Samsa GP, Weinberger M, Goldstein LB, Bonito A, Witter DM, et al. Health status of individuals with mild stroke. Stroke. 1997; 28(4): 740-745.

[3] Bernhardt J, Zorowitz RD, Becker KJ, Keller E, Saposnik G, Strbian, D, et al. Advances in stroke 2017. Stroke. 2018; 49(5): 174-e199.

[4] Lopez AD, Mathers CD. Measuring the global burden of disease and epidemiological transitions: 2002-2030. Annals of Tropical Medicine \& Parasitology. 2006; 100(5-6): 481-499.

[5] Horstman AM, Beltman MJ, Gerrits KH, Koppe P, Janssen TW, Elich P, De Haan A. Intrinsic muscle strength and voluntary activation of both lower limbs and functional performance after stroke. Clinical physiology and functional imaging. 2008; 28(4): 251-261.

[6] Cengiz A. Effects of self-selected dehydration and meaningful rehydration on anaerobic power and heart rate recovery of elite wrestlers. Journal of physical therapy science. 2015; 27(5): 1441-1444.

[7] Choi, YK, Park YH, Lee JH. Effects of sio taping and Mcconnell taping on balance and walking speed of hemiplegia patients. Journal of physical therapy science. 2016; 28(4): 1166-1169.

[8] Lee MS, Paik IY, Kwak YS, No HT, Jin HE. The Effect of Kinesio Taping on Muscle Strength Improvement, Blood Fatigue Factors, Muscle Fatigue and Damage Index. Journal of Life Science. 2010; 20(6): 870-876.

[9] Seo TH, Go HM, Park JH, Kim YH, Kim TW, Park HS. Effects of Kinesio Taping Applied on the Ankle Instability to Range of Motion and Balance. The Journal of Korean Academy of Orthopedic Manual Physical Therapy. 2017; 23(1): 7-13.

[10] Madhavan S, Bishnoi A. Comparison of the Mini-Balance Evaluations Systems Test with the Berg Balance Scale in relationship to walking speed and motor recovery post stroke. Topics in stroke rehabilitation. (2017); 24(8): 579-584.

[11] Jung SH, Park DS, Kang TK, An SH. The Discriminant Analysis and Predictive Validity for Predictor of Falls using STS, TUG, FRT, MSL, BBS in Patients with Chronic Stroke. The Research Institute for Special Education \& Rehabilitation Science. 2017; 56(1): 327-345.

[12] Eltoukhy M, Asfour S, Thompson C, Latta L. Evaluation of the Performance of Digital Video Analysis of Human Motion: Dartfish Tracking System. International Journal of Scientific \& Engineering Research. 2012; 3(3): 1-5.

[13] Kim WI, Park YH, Sung YB, Nam CW. Effects of kinesio taping for ankle joint and anklefoot orthosis on muscle stimulation and gait ability in patients with stroke suffering foot drop. Advanced Science and Technology Letters. 2015; 116: 261-265.

[14] van Duijnhoven HJ, Heeren A, Peters MA, Veerbeek JM, Kwakkel G, Geurts AC, Weerdesteyn V. Effects of exercise therapy on balance capacity in chronic stroke: systematic review and meta-analysis. Stroke. 2016; 47(10): 26032610.

[15] Giaquinto S, Giachetti I, Spiridigliozzi C, Nolfe G. Quality of life after stroke in a rehabilitation setting. Clinical and Experimental Hypertension. 2010; 32(7): 426-430.

[16] Tse T, Douglas J, Lentin P, Carey L. Measuring participation after stroke: a review of frequently used tools. Archives of physical medicine and rehabilitation. 2013; 94(1): 177-192.

[17] Lubetzky-Vilnai A, Kartin D. The effect of balance training on balance performance in individuals poststroke: a systematic review. Journal of neurologic physical therapy. 2010; 34(3): 127-137.

[18] Lewek MD, Bradley CE, Wutzke CJ, Zinder SM. The relationship between spatiotemporal gait asymmetry and balance in individuals with chronic stroke. Journal of applied biomechanics. 2014; 30(1): 31-36. 